\title{
Effects of current on vortex and transverse domain walls
}

\author{
J. He, Z. Li and S. Zhang \\ Department of Physics and Astronomy, University of Missouri-Columbia, Columbia, MO 65211
}

(Dated: August 9, 2018)

\begin{abstract}
By using the spin torque model in ferromagnets, we compare the response of vortex and transverse walls to the electrical current. For a defect-free sample and a small applied current, the steady state wall mobility is independent of the wall structure. In the presence of defects, the minimum current required to overcome the wall pinning potential is much smaller for the vortex wall than for the transverse wall. During the wall motion, the vortex wall tends to transform to the transverse wall. We construct a phase diagram for the wall mobility and the wall transformation driven by the current.
\end{abstract}

PACS numbers:

\section{INTRODUCTION}

In a magnetic wire, there are two standard walls: transverse wall (TW) and vortex wall (VW). In a certain range of the wire width and thickness, both types of the walls are stable [1, 2]. Experimentally, one can create either type of the wall by applying a magnetic field in various directions 3, 3]. The dynamics of both walls driven by a magnetic field has been extensively simulated [ $[, 6]$. In general, the mobility of the vortex wall is smaller than the transverse wall, and at a large magnetic field, the vortex core tends to move toward the wire edge and may be annihilated at the sample boundary. Thus a transformation from vortex wall to transverse wall may occur. In this paper, we address the wall dynamics driven by a current. It would be interesting to see how the currentdriven wall dynamics qualitatively differs from the fielddriven wall dynamics. The present work is also motivated by recent experimental observations on the domain wall motion [4]. Particularly, Kläui et al. have quantitatively measured the correlation between wall mobilities and wall structures, and a transformation from vortex wall to transverse wall has been clearly identified.

\section{MODEL}

We start with the generalized Landau-Lifshitz-Gilbert equation which includes the recently derived spin transfer torques [7]:

$$
\begin{aligned}
\frac{\partial \mathbf{M}}{\partial t}= & -\gamma \mathbf{M} \times \mathbf{H}_{e f f}+\frac{\alpha}{M_{s}} \mathbf{M} \times \frac{\partial \mathbf{M}}{\partial t} \\
& -\frac{b_{J}}{M_{s}} \mathbf{M} \times\left(\mathbf{M} \times \frac{\partial \mathbf{M}}{\partial x}\right)-\frac{c_{J}}{M_{s}} \mathbf{M} \times \frac{\partial \mathbf{M}}{\partial x}
\end{aligned}
$$

where $\gamma$ is the gyromagnetic ratio, and $\mathbf{H}_{\text {eff }}$ is the effective magnetic field including the external field, the anisotropy field, the magnetostatic field, and the exchange field, $\alpha$ is the Gilbert damping parameter, $b_{J}=$ $P j_{e} \mu_{B} / e M_{s}, c_{J}=\xi b_{J}, P$ is the spin polarization of the current, $j_{e}$ is the current density along the length direction of nanowire, $\mu_{B}$ is Bohr magneton, and $\xi$ is a dimensionless constant which describes the degree of the nonadiabaticity between the spin of the nonequilibrium conduction electrons and local magnetization.

\section{CALCULATION PROCEDURE}

We have solved the above LLG equation by performing a routine micromagnetic simulation for a magnetic wire whose geometrical size is $2 \mu \mathrm{m}$ long (x-direction, also the current direction), $128 \mathrm{~nm}$ wide, and $8 \mathrm{~nm}$ thick. The grid size is chosen as $4 \times 4 \times 8 \mathrm{~nm}^{3}$. The magnetization at both ends is set to be along the $\mathrm{x}$-direction and direct inward to the wire; and we use free boundary conditions for other dimensions. Since the domain wall may move up to several micrometers in some cases to be discussed below, it is important to keep the domain wall far away from the ends. To reduce this end effects due to the finite length of the wire, we shift the domain wall to the center of the wire after every small displacement or time interval, corresponding to the geometrical structure of wire. Using the similar initial domain configurations as used in 1], these two types of walls will form and they are our initial wall configurations at $t=0$. Starting at $t=0$, a static spin torque or a static magnetic field is applied to the wall until the end of the simulation. The strength of the spin torque or the field is chosen in a certain range and the dynamics of the wall after $t>0$ is reported in the paper. In simulating the pinning potential, we choose a geometrical boundary roughness by periodically removing, for every 32 grids in the length direction, one grid from each edge in the direction of wire width $[8]$.

The material parameters are: the exchange constant $A=1.3 \times 10^{-6} \mathrm{erg} / \mathrm{cm}$, the anisotropy field $H_{K}=$ $0(\mathrm{Oe})$, the saturation magnetization $M_{s}=800 \mathrm{emu} / \mathrm{cc}$, the damping parameter $\alpha$ is 0.02 , the spin polarization $P=0.5$, and the non-adiabaticity factor $\xi=0.04$. 


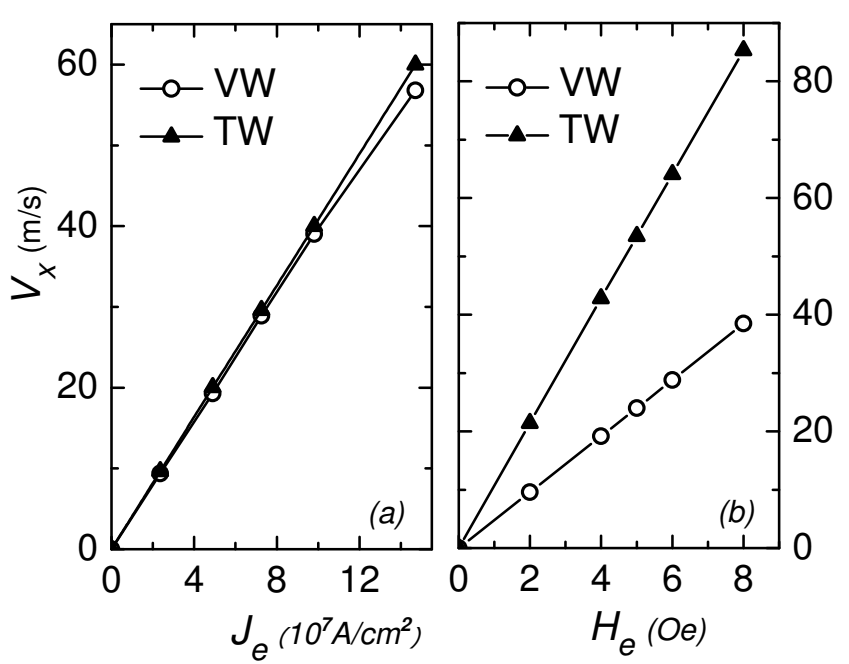

FIG. 1: Domain-wall steady state velocity $v_{x}$ as a function of (a) current $J_{e}$, and (b) field $H_{e}$. In (a) $v_{x}=-c_{J} / \alpha$ and in (b) $v_{x}=\gamma H_{e} W / \alpha$, both agree with the analytical result in (2)

\section{RESULTS}

\section{A. Defect-free domain wall motion}

A simplest case is a defect-free wire. In this case, the steady state wall velocity $v_{x}$ can be analytically derived,

$$
v_{x}=\frac{\gamma H_{e} W}{\alpha}-\frac{c_{J}}{\alpha}
$$

where $H_{e}$ it the external field and $W$ is the domain wall width defined as

$$
W^{-1}=\frac{1}{2 S M_{s}^{2}} \int d V\left(\frac{\partial \mathbf{M}}{\partial x}\right)^{2}
$$

where $S$ is the cross section area of the wire. The assumption used in deriving Eq. (2) is that the domain wall moves uniformly, i.e., $\mathbf{M}=\mathbf{M}\left(\mathbf{r}-v_{x} t \hat{\mathbf{x}}\right)$, see paper 9] of this proceedings. Thus, in the absence of the field, the steady velocity is $-c_{J} / \alpha$, independent of the wall structure [10]. In the presence of the field, however, the velocity depends on the wall width defined in Eq. (3). The vortex wall has a smaller effective width compared to the transverse wall and thus the vortex wall has lower velocity [1]. In Fig. (1), we show the steady state velocity for the vortex wall and the transverse wall. The wall velocity is well fitted by Eq. (2).

However, the uniform motion is expected to break down even for the defect-free wire when the external field or current density is large. For the vortex wall, when a current/field is turned on, the wall moves along the wire, and at the same time the vortex core also tends to move perpendicular to the wire. A restoring force [10] may stop this perpendicular movement and keep the vortex core inside the wire only when the current/field is sufficiently small. In this case, the wall motion remains steady and the velocity is given by Eq. (2) as shown in Fig. (1). The dynamics of the vortex wall at a higher current/field will be discussed in next section. For the transverse wall, if one applies a larger current/field, the uniform motion becomes unstable. In fact, the wall will constantly deform during its motion and the velocity is highly non-uniform, known as Walker's breakdown 12]. In the film we consider here, we choose the applied field and current far below the critical values for Walker's breakdown. We do not intend to describe the complicated dynamics after the breakdown in this paper.

\section{B. Domain wall motion in the presence of defects}

While there are many types of defects to pin a domain wall, we restrict our discussion on the defect induced by the roughness on the edges of the wire width. Specifically, we periodically remove one grid at each edge as described above in otherwise a defect-free wire.

Let us start with the transverse wall. When a current is applied, the domain wall begins to move and in the meanwhile the wall width shrinks. The wall shape remains similar to the original one. If the current is small, the wall eventually stops. The displacement of the center of the wall is proportional to the current density and the magnitude could be as large as several tens of nanometers. The terminal average velocity is zero. When the current exceeds a critical value $J_{t_{1}}$, as shown in Fig. (2a), the domain wall is able to overcome the pinning and can sustain a significant velocity, which is oscillating corresponding the defect positions (not shown here). In fact, the average velocity are smaller but still close to $-c_{J} / \alpha$ once the current density is larger than the critical current. It is understood that part of the effect of the defects has been compensated by the deformation of the domain wall and the average velocity has not been reduced significantly.

One interesting feature of the transverse wall is that the wall velocity as a function of the current is hysteretic. If the wall reaches the critical current, $J_{t_{1}}$, the wall begins to move with an average velocity, shown in Fig. (2a). Then after the wall is virtually depinned $\left(J \geq J_{t_{1}}\right)$ and a uniform wall velocity is established, one begins to reduce the current. It is found that the domain wall remains to have a finite velocity even if the current density has reduced to a value lower than $J_{t_{1}}$. One needs to further reduce the current density until reaching a second critical current, $J_{t_{2}}$, in order to completely stop the domain wall, see Fig. (2a). The hysteretic feature may be qualitatively understood in terms of the competition between the wall kinetic energy and defect potential [9]. When the current density is reduced from a higher value, the velocity of the wall remains large and it would be difficult for defects to pin the wall. On the other hand, when we increase the current density from a smaller value, the wall is originally 

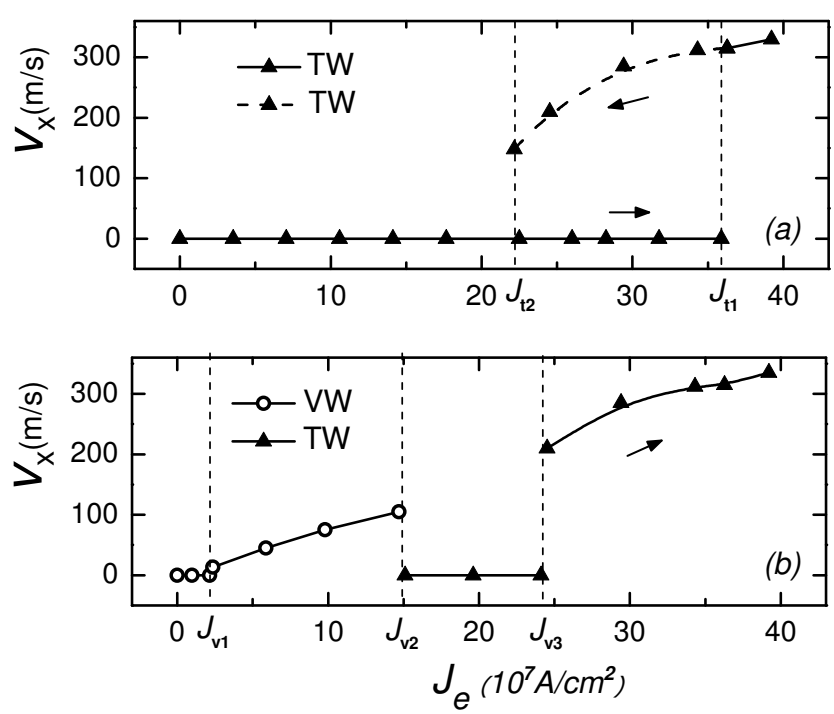

FIG. 2: The dependence of average domain-wall velocity on the current and wall structures. In (a), solid line shows the velocity of transverse wall by applying a current from the static state $\left(v_{x}(0)=0, J_{e}(0)=0\right)$; dashed line represents the velocity by applying a reduced current from a depinned state $\left(v_{x}(0) \neq 0, J_{e}(0)=J_{t_{1}}\right)$. In (b) the open circle line shows the average velocity of vortex wall, which depins at $J_{v_{1}}$ and transforms to a transverse wall at $J_{v_{2}}$; the up-triangle line represents the velocity of the transverse wall after the transformation: upon $J_{v_{3}}$, the transformed wall keeps the same velocity as in (a) with corresponding currents.

at zero velocity and thus the pinning is more effective.

The motion of the vortex wall is far more complex than that of transverse wall, because the transformation happens when the applied current/field is high. As in the transverse domain wall, one needs a critical value of the current to maintain a finite terminal velocity with vortex core inside the wall. However, the most interesting feature is that the critical current for the vortex wall, $J_{v_{1}}$, is far smaller than $J_{t_{1}}$, i.e., the vortex wall is less sensitive to the defect potential. As shown in Fig. (2b), the vortex wall begins to move at a current density an order of magnitude smaller than that for the transverse wall.
This is somewhat understandable because the effects of edge roughness on a vortex wall is quite small. In the previous work 13, it has been shown that the similar defect may trap the domain wall when being present at the center of vortex core, and it has almost no effects to the wall if staying at the edge.

When the current continues to increase, the average velocity of the wall proportionally increases. In the meanwhile, the center of the vortex core moves closer to the edge. At a second critical $J_{v_{2}}$, the vortex reaches the boundary and the vortex wall is transformed into a transverse wall. Because the critical current for the transverse wall $J_{t_{1}}$ is much larger, the wall motion stops. These features seem to agree with the experimental observation [4]. If the applied current is further increased upon to the third critical value $J_{v_{3}}$ which is smaller than $J_{t_{1}}$, the wall can still keep a finite velocity after the transformation is completed. And this average velocity just equals to the terminal average velocity that the transverse wall may sustain after the current is reduced from higher level, i.e. $J_{t_{1}}$ in Fig. (2a). It is also explained as the hysteretic feature of the transverse wall we described above: the kinetic energy gained before the wall transformation helps the transformed wall sustain a significant velocity.

\section{CONCLUSIONS}

We have investigated the domain wall dynamics for vortex and transverse walls by using the spin torque model. Interestingly, while the terminal velocity is independent of the wall structure for a steady wall motion in defect-free wire, the depinning current of the vortex wall is an order of magnitude smaller than the transverse wall in the presence of defects. However, the vortex wall is not as stable as the transverse wall during its motion. The vortex core may be annihilated at the edges and the transformation to the transverse wall occurs. These conclusions seem to explain the experimental observation [4]. This work is supported by NSF-DMR-0314456 and the MU research board.
[1] R.D.McMichael, M.J.Donahue, IEEE Trans. Magn. 33 4167 (1997).

[2] Y. Nakatani, A. Thiaville, J. Miltat, J. Magn. Magn. Mater. 290-291 750 (2005).

[3] A. Yamaguchi, T. Ono, S. Nasu, K. Miyake, K. Mibu, and T. Shinjo Phys. Rev. Lett. 92, 077205 (2004).

[4] M. Kläui, P.-O. Jubert, R. Allenspach, A. Bischof, J. A. C. Bland, G. Faini, U. Rüdiger, C. A. F. Vaz, L. Vila, and C. Vouille, Phys. Rev. Lett. 95, 026601 (2005).

[5] D. G. Porter, M. J. Donahue, J. Appl. Phys. 95, 6729 (2004).

[6] A. Thiaville, J. M. García, J. Miltat, J. Magn. Magn.
Mater. 242-245 1061 (2002).

[7] S. Zhang and Z. Li, Phys. Rev. Lett. 93, 127204 (2004).

[8] Jason Gadbois, Jian-Gang Zhu IEEE Trans. Magn. 31 3802 (1995).

[9] Z. Li, J. He and S. Zhang, (unpublished).

[10] A. Thiaville, Y. Nakatani, J. Miltat, and Y. Suzuki, Europhys. Lett. 69, 990 (2005).

[11] J. He, Z. Li and S. Zhang, (unpublished).

[12] N. L. Schryer and L. R. Walker, J. Appl. Phys. 45, 5406 (1974).

[13] Takashi Komine, et al., J. Appl. Phys. 78, 7220 (1995). 